\title{
Journal of Clinical Research \& Bioethics
}

\section{Expanding Patient Population and Medical Malpractice}

\section{Richard Boudreau}

Bioethics Institute, Loyola Marymount University, CA, USA

"Corresponding author: Dr. Richard Boudreau, Bioethics Institute, Loyola Marymount University, CA, USA, Tel: 310-822-6161; E-mail: rboudreaumdjd@aol.com

Received date: June 6, 2017; Accepted date: February 20, 2018; Published date: February 28, 2018

Copyright: @2018 Boudreau R. This is an open-access article distributed under the terms of the Creative Commons Attribution License, which permits unrestricted use, distribution, and reproduction in any medium, provided the original author and source are credited.

\section{Editorial}

One of the inevitable consequences of the Affordable Care Act is that millions of Americans who did not have access to health care in the past may be seeking care and physicians and medical facilities will have to address the needs of growing patient populations. From a physician standpoint, this growing population poses a number of issues, including care management, increasing workforce needs, increasing costs, and the potential for an increased number of malpractice lawsuits. This last issue, the problem of medical malpractice and the increase in the cost of malpractice insurance, has many physicians debating the viability of their practices in light of expanding costs.

One of the immediate assumptions is that a growing patient population will result in an increased number of malpractice claims. Malpractice claims more frequently extend from the treatment of patients requiring significant levels of care and often reflect the impacts of a lack of primary care that results in the onset of illness. In assessing the views related to both malpractice and increasing patient populations, physicians also need to balance off the cost savings that may result from patients obtaining preventative care, living healthier lifestyles, and not requiring complex treatments that are more likely to foster malpractice claims.

Obama's health care reform measures were designed to address the needs of the generally underserved in this country, including a large population of people who do not have access to health care services on a regular basis. The uninsured and underinsured in this country were the basic focus of the reform measures, with the belief that health care costs could be reduced significantly by implementing methods by which health care services could be paid and individuals could seek preventative care. The measures were implemented in order to increase access to quality health care while reducing costs, but the lack of a clear directive for reducing medical malpractice costs left physicians feeling like they were left out of the reform process. Physicians have called for a number of legislative measures, including reductions in liability limits as a means of addressing the financial costs of healthcare across the board. Excessive awards, high costs of premiums for malpractice insurance, and identified high-risk populations have influenced the decision-making of many physicians, especially in light of healthcare reform changes.

Though reform measures have been proposed, researchers have indicated that physicians still fear the impacts of malpractice claims, including claims that can alter their capacity to continue their practice. The Patient Protection and Affordable Care Act of 2010 (PPACA) will have a significant impact on the decision-making of many practicing physicians. The Act is likely to affect the coverage of about 32 million Americans, resulting in millions of added patients who require services. If all other variables remain unchanged, an influx of patient populations would result in an increase in the number of malpractice claims that are made and this would create a considerable burden to practicing physicians. Medical malpractice claims more frequently arise from surgical procedures or more complex treatments, but medical errors can occur at every level of care. One of the gaps in health care reform is to reform medical malpractice laws, creating some response to the issue before the problem fully manifest. Rothstein maintained that there is an increase in populations will lead to an increase in injured patients, and even balancing for potential improvements due to preventative care, physician errors leading to malpractice claims may not significantly decrease. Attempts to predict the behavior of injured patients in the new healthcare environment would be impossible to assess, but there is some evidence that these behaviors would not change significantly. The underlying reason is that regardless of the type of injury or the reason for the injury, people seeking malpractice lawsuits generally have reasons to attempt to sue for monetary damages that can include the need for money for longterm care; the need for continued medical care, the desire to deter future errors, and the need to mitigate for lost earning potential and lost livelihood. Often, patients have reported the decision not to file a malpractice claim even when admitted medical mistakes have occurred, based on a number of factors, including how they were informed about the error.

Another significant factor that may help to reduce concern about medical malpractice in a growing population is that national statistics suggest that malpractice cases have decreased in the last decade. This means that the number of payments made for physicians by their malpractice insurers have decreased, even though some findings suggest the overall payout per case has increased. Some of the factors that have generally led to a reduction in the number of claims include an increased diligence to reduce medical errors and increased preventative care services, which lead to fewer cases of long-term problematic health issues that can result in complicated treatments and surgeries in which medical errors are more likely to occur. In some cases, though, the number of case filings has decreased because physicians are less willing to work in areas and with populations that are at higher risk of filing medical malpractice claims. These findings suggest a need to consider broader reaching legislative changes that reduce the claims or reduce the cost of claims in order to maintain the directive for access to care inherent in the PPACA.

A major issue noted by physicians is that while health care reform has focused on methods of reducing costs while improving services, medical liability has gone relatively unaddressed, even though this is one of the major issues impacting costs. There is a "striking contrast to its prominence in previous federal health policy debates," and the malpractice insurance "crisis" which has led to expanding costs across the board. The call for linking medical liability reform with health care reform is based on the belief that these two issues are inextricably linked and that physicians who are responsible for caring for the growing population of people accessing health care should be able to 
manage their costs more effectively through reductions in liability insurance payments.

Specific reform measures have been proposed, including disclosureand-offer programs, administrative tribunals, and "safe harbor" options. Disclosure-and-offer programs are one approach to reform for malpractice that has fueled considerable debate. These programs are designed to support transparency and allow physicians to identify medical errors that have occurred while also providing a settlement offer in order to reduce the need for expensive lawsuits and protracted processes that result in exorbitant claims. The advantages of this kind of system is that it would promote transparency, improve reporting of medical errors, and reduce the volume of lawsuits, while also reduce the adversarial nature of the malpractice process. The disadvantages is that this type of program would probably be opposed by trial lawyers because it would reduce their role, and it would involve risk for health care providers because of the immediacy at which providers would have to inform their patients of the errors that occurred and the reasons for those errors. In addition, these programs have not had proven outcomes in relation to the program's success in reducing costs.

Another type of reform is the use of administrative or specialized tribunals, rather than a court-based legal process, which would reduce the costs involved in litigation. The advantages include an increase in the predictability of the process and the use of specific guidelines designed to reduce conflicting testimony. Because the tribunal would be conducted by medical professionals, it would place the error within a context and evaluation process that would improve understanding and reduce costs. At the same time, this type of approach may also be opposed by trial attorneys who would lose work, and it might be opposed by patient groups who may feel that this type of process unfairly supports the physician. Subsequently, this type of procedure might also be brought into question on the grounds of its constitutionality in terms of the right of the individuals to the trial process.

Finally, researchers have outlined the claims of "safe harbors" in which physicians who adhere to evidence-based practices can maintain the value of this kind of knowledge. This is perceived as a method of promoting physician dedication to research and practices of evidencebased care and could result in improved outcomes relative to the costs of malpractice suits. The disadvantages are that physicians may not use this kind of approach and costs may continue to skyrocket. Of the three potential areas of reform, this is the least likely to show immediate benefits in reducing the cost for a growing patient population.

Though states like Texas and California have applied reform measures to issues of medical malpractice, and have had some success in capping the costs involved, there are issues with doing this on a state-by-state level. Specifically, if some states implement malpractice liability reform, including caps on liability levels as a means of increasing the number of doctors who work in that state, they may actually increase the risk of malpractice by attracting high risk physicians. In other words, state-by-state reforms of liability insurance, including caps for malpractice suits, can result in a movement of highrisk doctors to states where reform measures have been put into place.
When addressing the needs of a growing patient population in specific areas, states like Texas have implemented reform to address this growing population. This seems like a lose-lose proposition, though, with increasing patient numbers and increasing high-risk doctors being matched together, thereby increasing the overall chances that malpractice will occur. Researchers have maintained that there is a statistically significant increase in the number of high-risk physicians in states that have implemented malpractice liability reforms. This is one of the underlying reasons that physicians have championed liability and malpractice reform measures at a national level.

There are two essential questions that must be considered when evaluating the call for malpractice reforms and the issue of a growing population of patients seeking health care services under the PPACA: 1. Does research support a link between the increased population and increased malpractice suits? 2. If reform is necessary, should it be applied at a state or national level? These two questions are at the crux of the debates and underscore the need for greater discourse on this issue.

First, the assumption cannot be made or easily supported that simply because there will be increased numbers of patients nationwide, that there will be an increase in the number of malpractice cases. In fact, increasing use of preventative medicine and the decreased need for interventive and surgical procedures could continue to support the decline in malpractice suits that has already been noted in the research. Second, even if reform measures are perceived as a necessary part of maintaining a lower average of malpractice claims per capita, there is generally little consensus about how this type of reform should be implemented. States like Texas have introduced tort reform measures and methods of capping malpractice suit outcomes, but these efforts may result in more claims, rather than fewer. Critics argue that in the absence of a federal mandate for malpractice reform, states with reform measures in place will see an influx of high-risk physicians and potentially have to bear the brunt of increasing malpractice suits.

Clearly, there is still need for additional discourse and research into the issue, but the flawed assumption that the PPACA will immediately result in increased malpractice suits and increased costs for care may never come to fruition. In fact, it is likely that over the next decade, malpractice suits may continue to decrease and this may correspond with the increasing access to preventative care services. In the meantime, physicians and health care facilities can make their own decisions about implementing methods to reduce malpractice, including individualized reform measures. Physicians and health care facilities, for example, can implement disclosure and offer approaches in which physicians are encouraged through administrative directives to disclose medical errors when they occur and work with internal counsel or hospital administration to determine what kind of offer can be utilized as a means of reducing the potential for malpractice suits. Assessing how this kind of approach can be introduced would be an essential part of hospital/primary care office planning. Though reform in medical malpractice was not a part of the current PPACA, the identification of the potential problem and the introduction of debate may serve as a foundation for additional national reforms in the future. 